\title{
Universal Mean Atomic Number curves for EPMA calculated by Monte Carlo simulations
}

Aurélien Moy ${ }^{1}$, Anette von der Handt ${ }^{2}$, John Fournelle ${ }^{1}$, William Nachlas ${ }^{1}$ and John Donovan ${ }^{3}$

${ }^{1}$ Department of Geoscience, University of Wisconsin-Madison, United States, ${ }^{2}$ Department of Earth and Environmental Sciences, University of Minnesota, Minneapolis, Minnesota, United States, ${ }^{3}$ CAMCOR, University of Oregon, United States

Electron Probe Microanalysis (EPMA) requires the acquisition of characteristic X-ray intensities of elements of interest, which are superimposed upon the background (also called continuum or bremsstrahlung). These background counts must be removed for accurate quantitation, usually by measuring two off-peak channels. An alternative approach, the Mean Atomic Number (MAN) background calculation method, was developed in 1970s. This technique takes advantage of Kramers' law: the generated continuum intensity scales monotonically with the (mean) atomic number of the target. Here, an empirical curve of background intensity versus MAN is generated via measurements at a specific characteristic X-ray peak position for a desired element on a series of standards that do not contain the analyte of interest [1,2]. After a correction for continuum absorption upon the emitted X-rays, we can assume that the MAN curve is a smooth function of the mean atomic number and can easily be used to determine the continuum intensity of a particular material, given its mean atomic number.

The MAN background method offers several benefits: time savings by only acquiring peak intensities, less spectrometer wear, and elimination of vibrations from spectrometer movement that can be problematic at high spatial resolution operation. The MAN technique is especially useful for improving precision in trace element analysis [3] and analyzing beam sensitive materials as beam exposure time is reduced. Quantitative X-ray mapping especially benefits from the use of the MAN method [4]. However, creating the necessary MAN curves can be problematic, if appropriate high purity standards are not available, and/or high precision trace measurements (e.g., 5-10\%) are desired.

During our EPMA technique research the question arose: could we extract theoretical continuum intensities and generate precise theoretical MAN curves utilizing PENELOPE/PENEPMA Monte Carlo simulations [5]? The answer is yes, provided a comprehensive data treatment is applied. The emitted continuum intensity (experimental and Monte Carlo data) needs to be corrected for isotropic and anisotropic absorption as well as for electron backscattering effects. It is critical to apply these corrections because different matrix compositions with different elements may have similar mean atomic numbers but can present strong differences in absorption coefficients and electron backscattering factors, leading to different emitted continuum intensities. This allows us to validate the accuracy of X-ray emission models used to produce the MAN curves as well as the model used to calculate the mean atomic numbers.

We used the PENEPMA code to calculate continuum intensities emitted from single element and multicomponent materials and performed calculation for all elements of the periodic table and 43 compounds commonly found in EPMA labs. The calculated intensities were corrected for absorption and electron backscattering effects using the model of Small et al. [6]. Donovan et al. [7,8] showed that the traditional mass weight fraction averaged atomic number does not always produce adequate results because isotopic measurements have demonstrated that mass does not significantly affect continuum (or backscatter) production [7,8]. They proposed a nonlinear electron-fraction weighted averaging expression, with an exponential term of 0.7 (or 0.8 ) determined experimentally, producing better results for most 
compounds used in their study [7,8]. We found that best results were obtained when using mean atomic numbers calculated using the electron-fraction weighted averaging expression [9] with an exponent of 0.6:

$$
\bar{Z}=\frac{\sum_{i=0}^{N} a_{i} \times Z_{i} \times Z_{i}^{x}}{\sum_{i=0}^{N} a_{i} \times Z_{i}^{x}}
$$

where $\mathrm{a}_{\mathrm{i}}$ is the atomic fraction of element $\mathrm{i}, \mathrm{Z}_{\mathrm{i}}$ is the atomic number of element $\mathrm{i}$ and $\mathrm{x}=0.6$ is the exponent. The index i runs on all $\mathrm{N}$ elements constituting the material.

Fortunately, because the continuum intensity is measured at the same energy for all the standards, the spectrometer detection efficiency remains the same. Hence, for a specific X-ray energy, MAN curves acquired on different spectrometers have the same shape as the theoretical ones. By normalizing these curves to the continuum intensity measured on a common standard (e.g., $\mathrm{Al}_{2} \mathrm{O}_{3}$ ), universal MAN curves can be obtained and used on any electron microprobe and any WD spectrometer. Figure 1 shows the good agreement between calculated continuum intensities with experimental measurements acquired on different instruments and different spectrometers.

We developed a user-friendly program to automatically extract continuum intensities at a given X-ray energy from previously run Monte Carlo simulations. Simulation data is corrected for absorption and backscattering effects, normalized to a given standard, and fit with a polynomial function. This program allows us to generate a library of universal MAN curves that can theoretically be used on any instrument (with a takeoff angle of 40 degrees) as long as the same standard material is used to normalize the curve. As an example, Figure 2 compares measured and calculated continuum intensities (not corrected) corresponding to the $\mathrm{Mg} \mathrm{K} \alpha \mathrm{X}$-ray line energy in a range of $\mathrm{Mg}$-free materials and $\mathrm{MgO}$. The calculated values have been uncorrected from the absorption and electron backscattering effect using the theoretical MAN curve. The background intensity for $\mathrm{MgO}$ was determined by recording the spectrum around the $\mathrm{Mg} \mathrm{K} \alpha$ line and interpolating the background under the peak. Satisfactory results were obtained for all samples except for the Si standard where the continuum intensity is overestimated by $27 \%$ by the calculations (a longer counting time might improve this result).

By providing a simple and comprehensive approach to determine X-ray continuum intensities at any Xray energy of interest, our program provides a powerful tool to streamline the MAN background methodology for quantitative X-ray microanalysis. 

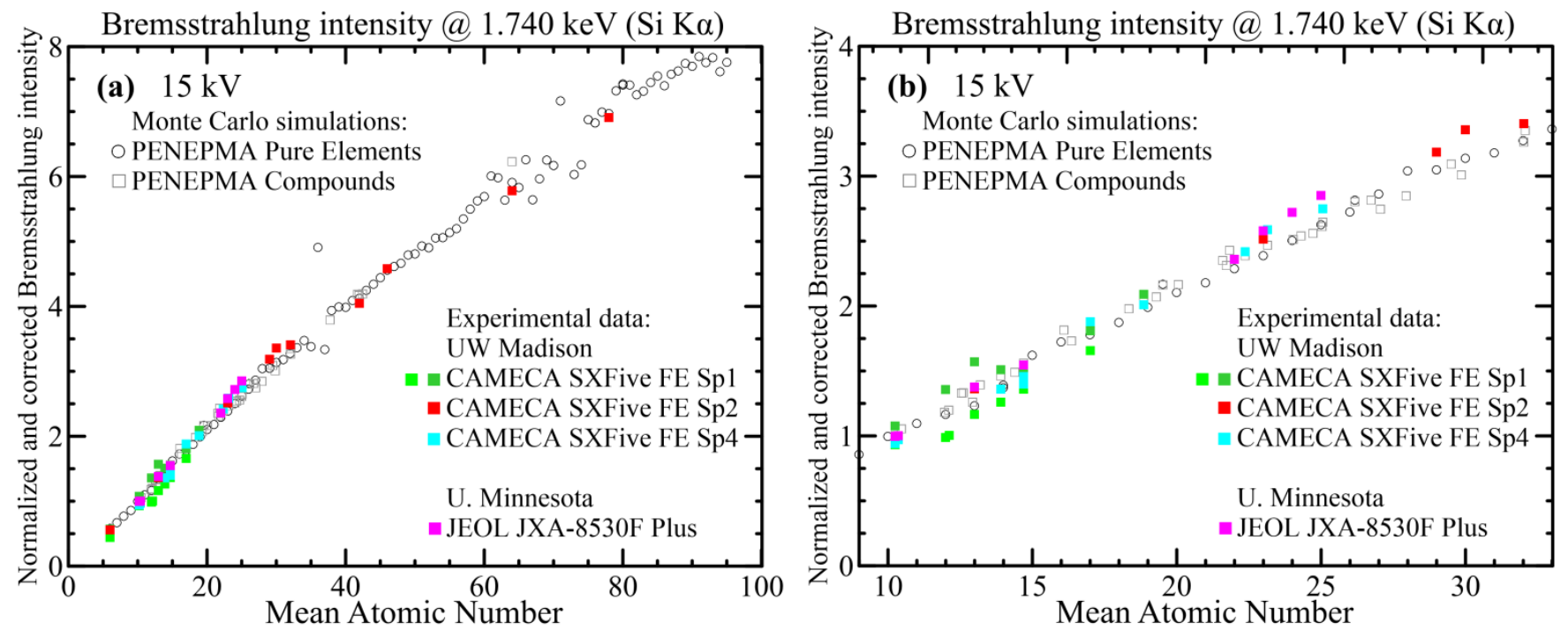

Figure 1. Corrected and normalized (relative to Al2O3) bremsstrahlung intensities corresponding to the Si Ka X-ray line at $15 \mathrm{kV}$. Experimental and Monte Carlo simulation data fall on the same curve. (b) is a zoomed in portion of (a) with MAN ranging from 10 to 30, corresponding to most materials found in geology.

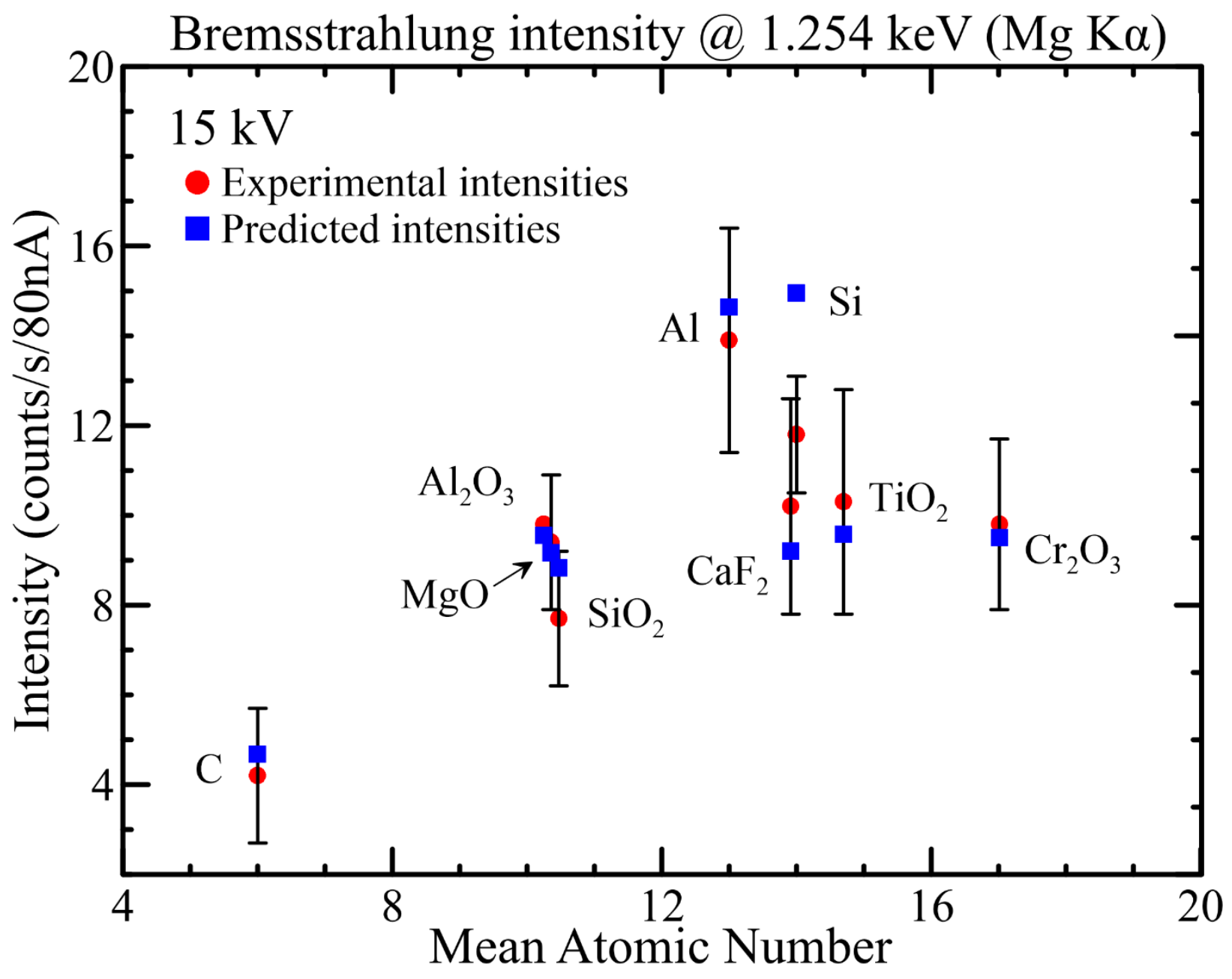


Figure 2. Bremsstrahlung (not corrected) intensity at the $\mathrm{Mg} \mathrm{K} \alpha \mathrm{X}$-ray energy measured (by WDS) and predicted (using the electron-fraction weighted averaging expression with an exponent of 0.6) on nine $\mathrm{Mg}$-free standards and one $\mathrm{MgO}$ standard at $15 \mathrm{kV}$. Good agreement was found between the calculations and the measurements (experimental error bars $=1 \sigma$ statistical uncertainty).

\section{References}

[1] Merlet C. and Bodinier J-L. (1990) Electron microprobe determination of minor and trace transition elements in silicate minerals: A method and its application to mineral zoning in the peridotite nodule PHN 1611. Chemical Geology, 83:55-69

[2] Donovan, J.J. and Tingle, T.N. (1996) An improved mean atomic number background correction for quantitative microanalysis. Microscopy and Microanalysis, 2:1-7.

[3] Donovan, J.J., Singer, J.W., and Armstrong, J.T. (2016) A new EPMA method for fast trace element analysis in simple matrices. American Mineralogist, 101:1839-53.

[4] Barkman, J.E., Carpenter P., Zhao, J-C., and Donovan, J.J. (2013) Electron microprobe quantitative mapping vs defocused beam analysis. Microscopy and Microanalysis, 848-9.

[5] Llovet, X., and Salvat, F. (2016) PENEPMA: A Monte Carlo programme for the simulation of X-ray emission in EPMA. IOP Conference Series: Materials Science and Engineering, 109.

[6] Small, J.A., Leigh, S.D., Newbury, D.E., and Myklebust, R.L. (1987) Modeling of the bremsstrahlung radiation produced in pure-element targets by 10-40 keV electrons. Journal of Applied Physics, 61, 459469.

[7] Donovan, J.J., and Pingitore, N.E. (2002) Compositional averaging of continuum intensities in multielement compounds. Microscopy and Microanalysis, 8, 429-436.

[8] Donovan, J.J., Pingitore, N.E. and Westphal, A. (2003) Compositional averaging of backscatter intensities in compounds. Microscopy and Microanalysis, 9, 202-215.

[9] Donovan, J.J., Fellowes, J., and McMorran, B. (2019) Average Atomic Number and Electron Backscattering in Compounds. Microscopy and Microanalysis, 25, 2314-2315.

[10] Support for this research came from the National Science Foundation: EAR-1337156 (JHF), EAR1554269 (JHF), EAR-1849386 (JHF) and EAR-1849465 (AVDH). 